\title{
String fluid dynamical models in the Einstein-Cartan theory
}

\author{
Larry L Smalley $\dagger$ and J P Krisch $\ddagger$ \\ $\dagger$ Department of Physics, University of Alabama in Huntsville, Huntsville, AL 35899, USA \\ ¥Department of Physics, University of Michigan, Ann Arbor, MI 48109, USA
}

Received 17 November 1997, in final form 27 October 1998

\begin{abstract}
Using the general, energy-momentum tensor for a dynamical, string fluid developed from the Ray-Hilbert variational principle, physically motivated applications to Riemann-Cartan spacetime are given within the framework of the Einstein-Cartan theory. The string density is considered for the cases when it is or is not a thermodynamical variable of the fluid. Solutions are given for both cases and compared with other models in both general relativity and the EinsteinCartan theory. The use of a string fluid as a contributer to galactic halo density is also discussed.
\end{abstract}

PACS numbers: $0440 \mathrm{~N}, 0450,0570,1127,9880 \mathrm{C}, 9880 \mathrm{H}$

\section{Introduction}

Fluid atmospheres and environments for relativistic objects are intrinsic to parameter-extended Schwarzschild sources. A radiation atmosphere for black holes was developed by Vaidya [1] by allowing the Schwarzschild mass parameter to be a function of retarded time. A black hole Hawking atmosphere has been discussed by 't Hooft [2,3]. It was shown recently [4] that allowing the Schwarzschild mass parameter to be a function of the radial coordinate as well as retarded time generated an anisotropic string fluid atmosphere in addition to the Vaidya null fluid. With the apparent connections between string states and black hole entropy $[5,6]$, string fluid atmospheres have gained increasing importance. Letelier, using a slightly modified static Schwarzschild source, discussed the effects of a simple atmosphere of dusty strings [7] and strings with tension [8,9]. Soleng [10], extending these ideas, pointed out that a string fluid could provide a mechanism explaining the observed galactic rotation curves. Some early descriptions of the dark matter halos that modelled the observed data, predicted a density dropping like $r^{-2}$. Higher-resolution simulations seem to indicate that the density has a varying dependence, going like $r^{n}$, with $n=-1$ close to the centre and $n=3$ to 4 for large radii $[11,12]$ contributions. On a galactic scale, the average density still goes like $r^{-2}$.

The Lagrangian used in many string fluid calculations is an extension of the string formalism developed for high-energy applications by Nambu [13], Nielson [14] and Susskind [15]. Sherk's review [16] discusses Nambu's suggestion that the action for a free string should be proportional to the area of the string worldsheet, generalizing the worldline action for a relativistic point particle. Stachel [17] elaborated on this suggestion and developed a stressenergy content for a fluid of strings that generalized the usual perfect-fluid stress-energy content. For a dust fluid, the stress-energy is

$$
T_{i j}=\rho U_{i} U_{j}
$$


where $U_{i}$ is the fluid velocity and is taken to be the timelike member of a tetrad, $a^{\mu i}$. Greek indices are anholonomic and run from 1 to 4 . They are raised and lowered with a Minkowski metric $(1,1,1,-1)$. Latin indices run from 0 to 3 and label the holonomic coordinates. They are raised and lowered with the metric tensor for the spacetime, $g_{i j}$ with signature $(-,+,+,+)$. Since the fluid velocity describes motion by an element of the fluid along its worldline, Stachel [17] generalized the dust stress-energy to string fluids by writing

$$
T_{i j}=\rho \Sigma_{i k} \Sigma_{j}^{k}
$$

where $\Sigma_{i j}$ is a simple, surface-forming bivector which spans the two-dimensional (2D) timelike worldsheet of the string. This stress-energy tensor has been used extensively by Letelier [7-9], Soleng [10] and others [18] in developing string fluid solutions to the Einstein field equations.

The string bivector is similar in structure to the bivector $S_{i j}$, describing the spin density in a spin fluid. The spin bivector, assumed to lie along the $\mu=3$ direction, is written in terms of a spin module function, $\kappa$, the conserved fluid density $\rho$ and the tetrads

$$
S_{i j}=\rho \kappa\left(a_{i}^{1} a_{j}^{2}-a_{j}^{1} a_{i}^{2}\right)
$$

while the string bivector can be written as

$$
\Sigma_{i j}=\rho \lambda\left(U_{i} a_{j}^{3}-U_{j} a_{i}^{3}\right) .
$$

The form of equation (4) follows closely the form of the spin bivector developed by Halbwachs [19] and therefore the description of a string fluid energy density will use the parallel description of the velocity matrix used in discussions of fluid dynamics in continuum mechanics [20]. Absorbing the density and string module function into a string vector, $S_{i}$, this can be rewritten as

$$
\Sigma_{i j}=-8 S_{[i} U_{j]} .
$$

Smalley and Krisch [21], noting this similarity, developed a string fluid Lagrangian that paralleled the spin fluid Lagrangian developed by Ray and Smalley [22, 23].

The matter Lagrangian for a spin fluid can be written as the sum of two terms. The first is the usual perfect-fluid Lagrangian

$L_{p f}=e\left\{-\rho[1+\epsilon(\rho, s)]+\lambda_{\mu v}\left(g_{i j} a^{\mu i} a^{v j}-\eta^{\mu \nu}\right)+\lambda_{2} \nabla_{i}^{*}\left(\rho U^{i}\right)+\lambda_{3} U^{i} X_{, i}+\lambda^{4} U_{i} s,{ }_{i}\right\}$

where the $\lambda$ 's are the Lagrange multipliers that enforce tetrad orthonormality, entropy conservation along the flow lines, continuity and particle number conservation, $e=\sqrt{-g}$ where $g$ is the determinant of the metric, and the 'star' derivative is given by $\nabla_{i}^{*}=\nabla_{i}+2 S_{i}$. (This same Lagrangian can be used in a regular spacetime with a Christoffel connection or in a Riemann-Cartan (RC) spacetime with a torsion connection requiring only that $\nabla_{i}^{*} \longleftrightarrow \nabla_{i}$.) A variation of the action for this Lagrangian alone produces a perfect-fluid stress-energy tensor [24],

$$
\left.T_{i j} \text { (perfect fluid }\right)=(\rho+p) U_{i} U_{j}+p g_{i j} .
$$

The second term in the complete spin fluid Lagrangian is the kinetic energy of the spin fluid written as the dot product of the spin density times the fluid angular velocity $\omega_{i j}$,

$$
K E_{\text {spin }}=\frac{1}{2} S^{i j} \omega_{i j}
$$

where

$$
\omega_{i j}=a_{i ; k}^{\mu} U^{k} a_{\mu j} .
$$

The action variation for the complete Lagrangian results in a spin fluid stress-energy tensor which can be used in either a general relativistic spacetime or a spacetime with torsion. 
Using the similarity between the structure of a spin fluid and a string fluid, a string fluid Lagrangian can be written down which also contains two terms. The first is just the perfectfluid contribution, equation (7). The second is written in analogy to the spin fluid. Comparing to equation (8) we write a string contribution to the Lagrangian as

$$
K E_{\text {string }}=\frac{1}{2} \Sigma^{i j} \omega_{i j}
$$

Some additional justification for this formulation is given by Nieto [25]. An action variation using the complete string fluid plus gravitational Lagrangian produces the field equations and allows the identification of the string fluid stress-energy content. The variations and the string fluid stress-energy content are described in [21]. While the formalism can be used in either a general relativistic space or a spacetime with torsion, there is a very surprising difference between the two cases. In a general relativity (GR) spacetime, the stress-energy involves the string function $S_{i}$. In a Riemann-Cartan (RC) spacetime, the stress-energy also involves the string function but, in addition, the variations identify the string function with the torsion vector [26] coming from the torsion contributions to the connection. The torsion vector has been identified with matter-radiation fields in Einstein-Maxwell calculations [27, 28]. Its variational identification with a string fluid parameter is significant since it provides a geometric link for the string function and also because the presence of torsion has a direct effect on the string fluid structure.

In the next section we briefly review the field equations obtained in [21]. In the last two parts of the paper we develop a solution to the field equations and discuss the modifications that torsion introduces.

\section{Field equations with torsion}

The Ray-Hilbert [27] variational method for a string fluid in an RC spacetime gives the following metric-field equation and the corresponding energy-momentum tensors

$$
G^{(i j)}-\nabla_{k}^{*}\left(T^{k i j}+T^{k j i}\right)=\kappa T^{i j} \text { (string fluid). }
$$

The star derivative would be the regular covariant derivative in a general relativistic spacetime. In a space with torsion, the star derivative [26] is

$$
\nabla_{i}^{*}\left(f_{j}\right)=f_{i ; j}+2 S_{i} f_{j}
$$

where the covariant derivative contains the usual additional torsional contribution to the connections and $S_{i}$ is the torsion vector which has been identified with the string function. $T^{k i j}$ is the modified torsion [26]. From the variations, the torsion field equation is

$$
T^{k i j}=\frac{1}{2} \kappa \Sigma^{k i} U^{j}
$$

As discussed in the introduction, the complete stress-energy tensor, $T_{i j}$ (string fluid), is the sum of two terms, a perfect-fluid stress-energy tensor and the string stress-energy tensor, given by

$$
T_{i j}(\text { string fluid })=T_{i j}(\text { perfect fluid })+T_{i j}(\text { string }) .
$$

For the case where the internal energy is not considered to be a function of the string variables, the string contribution is [20]

$$
\kappa T^{i j}(\text { string })=\nabla_{k}^{*}\left[U^{(i} \Sigma^{j k)}\right]+\nabla_{k}^{*}\left[U^{k} \Sigma^{m(j}\right] U^{i)} U_{m} .
$$

When the string variables are considered to be thermodynamic, the string stress-energy tensor becomes

$$
\kappa T^{i j}(\text { string })=U^{(i} \Sigma^{j) k} \dot{U}_{k}-\dot{U}^{(i} \Sigma^{j) k} U_{k}+\nabla_{k}^{*}\left[U^{(i} \Sigma^{j) k}\right]+\nabla_{k}^{*}\left[U^{k} \Sigma^{m(j}\right] U^{i)} U_{m} .
$$


From the variation, $\Sigma_{i j}$ can be written in terms of the string vector $S_{i}$

$$
\kappa \Sigma^{i j}=-8 U^{[i} S^{j]}
$$

where the torsion vector is

$$
S^{i}=-\frac{1}{4} \kappa \rho a^{3 i} .
$$

By comparison with the string vector, $S_{i}$, discussed in [29], the string and torsion vectors are related by $\kappa \mathcal{S}^{i}=16 S^{i}$. A direct consequence of equation (5) is that the torsion vector is a spacelike vector. This also implies that the spin vector is proportional to the fluid acceleration, $S_{i} \sim \dot{U}_{i}$.

In the applications described below, it is necessary to consider that the string variable, may or may not be a thermodynamic variable with respect to the second law. To distinguish the two cases a parameter $b$ is used where

$$
\begin{aligned}
& b=1 \text { variable not thermodynamic } \\
& b=2 \text { variable is thermodynamic. }
\end{aligned}
$$

With this parametrization, the string contributions from equations (15) and (16) can be combined in the form

$$
\begin{aligned}
\kappa T^{i j}(\text { string })= & 4\left\{-U^{i} U^{j}\left(S^{k}{ }_{; k}+b S^{k} \dot{U}_{k}+2 S^{k} S_{k}\right)+b \dot{U}^{(i} S^{j)}+2 U^{(i} \dot{S}^{j)}\right. \\
& \left.+2 U^{(i} S^{j)} U^{k}{ }_{; k}-S^{k} \nabla_{k}\left(U^{i} U^{j}\right)\right\} .
\end{aligned}
$$

\section{Applications}

\subsection{Fluid parameters}

As an application of the string fluid formalism in a Riemann-Cartan spacetime, a string fluid is considered in a static spherically symmetric spacetime describe by the metric

$$
\mathrm{d} s^{2}=-f(r) \mathrm{d} t^{2}+\frac{\mathrm{d} r^{2}}{f(r)}+r^{2} \mathrm{~d} \Omega^{2} .
$$

Considering only a radial string distribution

$$
S^{i}=(0, S, 0,0)
$$

the general fluid parameters can be written as

$$
\begin{aligned}
& \rho=-\frac{f^{\prime}}{r}+\frac{1-f}{r^{2}}+4 S^{\prime}-S\left(2 \frac{f^{\prime}}{f}-\frac{8}{r}\right)+\frac{4}{3} \frac{S^{2}}{f}+4 S(b-1)\left(\frac{f^{\prime}}{2 f}-\frac{2 S}{3 f}\right) \\
& p_{r}=\frac{f^{\prime}}{r}-\frac{1-f}{r^{2}}-2 S \frac{f^{\prime}}{f}+\frac{4}{3} \frac{S^{2}}{f}-4 S(b-1)\left(\frac{f^{\prime}}{2 f}-\frac{2 S}{3 f}\right) \\
& p_{\theta}=p_{\varphi}=\frac{f^{\prime \prime}}{2}+\frac{f^{\prime}}{r}+\frac{4}{3} \frac{S^{2}}{f}
\end{aligned}
$$

where $b$ is the thermodynamic parameter and primes indicate derivatives with respect to $r$. The usual equation of state for a spherically symmetric string fluid is

$$
\rho+p_{r}=0 .
$$

It is seen from equations (23) and (24) that the equation of state takes the form

$$
\rho+p_{r}=4 S^{\prime}-S\left(\frac{4 f^{\prime}}{f}-\frac{8}{r}\right)+\frac{8}{3} \frac{S^{2}}{f}
$$


and it is independent of the thermodynamic parameter, $b$. The inclusion of torsion and its identification with the string vector provides a family of possible models whose equation of state depends on the string function and $f(r)$. If we impose the string equation of state we can relate the string distribution function to $f(r)$ without knowing its explicit form. Imposing $\rho+p_{r}=0$, one finds the string distribution function is

$$
S^{r}=\frac{A f}{r(r-2 A / 3)} .
$$

\subsection{Choice of $f(r)$}

The choice of $f(r)$ has been made in a variety of ways. For example, Letelier [7] has discussed a solution

$$
f=1-h-\frac{2 M}{r}
$$

where $h$ models the presence of a black hole string atmosphere in general relativity. In general relativity, the equation for the transverse pressures is equation (25) with the $S^{2}$ term absent. Letelier's choice follows from requiring the transverse stress to be zero. This form of $f(r)$ was used in discussing the general relativistic version of the string fluid model discussed by Smalley and Krisch [29]. In discussing galactic dark matter, Soleng [10] used

$$
f=A-\frac{2 M}{r}+\frac{C}{r^{2 d}}
$$

where $d$ is the Tolman parameter [30]. This choice contains the $d=+1$ Reissner-Nordström solution, the $d=-1$ de Sitter point mass and $C=0, A=1-h$ is the Letelier solution. Soleng's $f(r)$ produces a gravitational acceleration which for small $d$ motivates the $r^{-1}$ gravity sometimes used to explain galactic rotation data $[11,12]$.

There are several possibilities for finding a solution for $f(r)$ to use in the presence of torsion. We could use the Letelier choice with our string distribution function, equation (28), and compare the results to the general relativistic string fluid [29]. We could use the form, equation (28), for the string distribution function and by requiring zero transverse stress, determine $f(r)$ from the field equations, equation (25). A third possibility is to choose $f(r)$ to be

$$
f(r)=1-\frac{2 M}{r+r_{o}} .
$$

This $f(r)$ is motivated by several results. Dark matter halos may have a very much richer structure than a simple $r^{-1}$ fall-off in acceleration would indicate. There seem to be three regions: a central core going as $r^{-1}$, and intermediate and outer regions where the density falls off more and more steeply [31].

The galactic halo dark matter density profile has been found in a number of parameter fits and simulations to be given by

$$
\rho(r)=\frac{b}{\left(r+r_{1}\right)\left(r+r_{o}\right)^{n}} .
$$

Using numerical $N$-body simulations, Navarro et al $[32,33]$ have studied this density profile with $r_{1}=0, n=2$. The simulations were performed in a variety of cosmological settings, including various Einstein-de Sitter universes. The density profile provided a good fit to dark matter halos for two decades in radius for galactic masses from about $3 \times 10^{11}$ to $3 \times 10^{15}$ solar masses. Their model gives an $r^{-3}$ asymptotic fall off in the outer region. Hernquist [34], used the same form with $r_{1}=0$ and $n=3$, giving a faster fall off, $r^{-4}$. These models were obtained 
without torsion. While this density profile is based on a specific set of metrics, modelling a torsional density function of the form of equation (32) through the field equations suggests that $f(r)$ should have the form (31). Also, strings are often considered as a possible dark matter candidate, and this form for $f(r)$ is also suggested by the string distribution function $S(r)$ given by equation (28). Because of the similarity in form between the string distribution function and $f(r)$, we could identify $r_{o}$ with $-2 A / 3$. Note that this makes the string density negative. We will explore all three possibilities and compare them.

3.2.1. The Letelier choice of $f(r)$. Using equations (28) and (29) in the field equations one finds an energy density and stresses

$$
\begin{aligned}
& r^{2} \rho=-r^{2} p_{r}=h+\frac{A}{(r-2 A / 3)}\left\{\frac{4 b M}{r}-\frac{4 A f(2 b-1)}{3(r-2 A / 3)}\right\} \\
& p_{\vartheta}=p_{\varphi}=\frac{4}{3} \frac{S^{2}}{f} .
\end{aligned}
$$

In general relativity, this same model has a string and stress-energy structure [29]

$$
\begin{aligned}
& S=\frac{S_{o} f}{r^{2}} \\
& r^{2} \rho=-r^{2} p_{r}=h+\frac{1}{4} b M S_{o} \\
& p_{\vartheta}=p_{\varphi}=0 .
\end{aligned}
$$

The major differences are a non-zero transverse stress which is entirely due to the torsion vector and the much richer density structure. The asymptotic behaviour of density and radial stress is $r^{-2}$ for both cases.

3.2.2. Determining $f(r)$ by requiring zero transverse stress. Using the string distribution function equation (28) in the field equation (25), one finds an equation for $f(r)$

$$
f^{\prime \prime}+\frac{2 f^{\prime}}{r}+\frac{8}{3} \frac{f A^{2}}{r^{2}(r-2 A / 3)^{2}}=0 .
$$

Defining $u=1 / r$ and $w=1-2 A u / 3$, this can be cast into the form

$$
\frac{\mathrm{d}^{2} f}{\mathrm{~d} w^{2}}+\frac{6 f}{w^{2}}=0
$$

easily solved by

$$
f(r)=C_{1}\left(1-\frac{2 M}{3 r}\right)^{3}+C_{2}\left(1-\frac{2 A}{3 r}\right)^{-2} .
$$

Asymptotically this can be cast into the Letelier form with the constants equal to

$$
C_{1}+C_{2}=1-h \quad C_{1}-\frac{2 C_{2}}{3}=\frac{M}{A} .
$$

3.2.3. Determining $f(r)$ from halo modelling. Assuming the form which might be associated with a halo, equation (31), we will write the energy density and compare it to the simulation forms,

$$
\rho=\frac{1}{r\left(r+r_{o}\right)^{2}}\left[\frac{4 M r_{o}(3 b-1)-3 r_{o}^{2}(2 b-1)}{r}-\frac{6 M r_{o}(3 b-1)}{r+r_{o}}\right]
$$


where $r_{o}=-2 A / 3$. Rewriting equation (40) gives

$\rho=\frac{1}{r^{2}\left(r+r_{o}\right)^{3}}\left\{r_{o}^{2}\left[4 M(3 b-1)-3 r_{o}(2 b-1)\right]-r r_{o}\left[2 M(3 b-1)+3 r_{o}(2 b-1)\right]\right\}$.

If one chooses $4 M(3 b-1)=3 r_{o}^{2}(2 b-1)$, the density profile form is that found by Hernquist but the density is negative,

$$
\rho=\frac{-6 M r_{o}(3 b-1)}{r(r+8 M / 3)^{3}} .
$$

This could possibly describe a structure with a negative mass black hole. Mann [35] has discussed such an object. It is not suggested by the halo models. The second choice that can be made in the density given by equation (41) sets the second term in the numerator to zero, so that

$$
2 M(3 b-1)=-3 r_{o}(2 b-1) .
$$

One finds two possible values of $r_{o}$, depending on $b$.

$r_{o}=-\frac{4}{3} M \quad$ for $\quad b=1 \Leftrightarrow$ string variables are not thermodynamic

$r_{o}=-\frac{10}{9} M \quad$ for $\quad b=2 \Leftrightarrow$ string variables are thermodynamic.

If this were describing an atmosphere around a black hole, the presence of the string atmosphere would tend to inflate the horizon. The density profile is

$$
\rho=6 M r_{o}^{2}(3 b-1) r^{2}\left(r+r_{o}\right)^{3} .
$$

This is again most similar to the Hernquist [34] density profile except it has an extra $r^{-1}$ fall off. If one considered the entire density described by equation 41 without restricting the parameters it is clear that there is a point at which the density vanishes,

$$
r=r_{o} \frac{4 M(3 b-1)-3 r_{o}(2 b-1)}{2 M(3 b-1)+3 r_{o}(2 b-1)} \text {. }
$$

This implies that there is a boundary in this string model which is not present in the Navarro and Hernquist models. If there is a singular object at the centre of the galaxy, one would not expect the density function to be valid past the horizon. The boundary in this model limits the effect of the strings to intermediate regions. One would not expect to see the effect of the dark matter strings at infinity.

\section{Conclusion}

Torsion can have a large influence on modelling outcomes. It is tempting to argue from the standpoint of a theorem [36] on the uniqueness of a symmetric connection in a Riemannian manifold (which is both torsion and non-metricity free) that this uniqueness is equivalent to the 'gauging' away of the torsion and non-metricity. If this is compared to electromagnetic theory with the invariance of the electric and magnetic fields under the gauging of the vector potential, one quickly notes that the gravitational field does not have the same invariant behaviour because of the different possible manifolds in the metric affine geometry. When considering spin fluids, for example, the spin energy enters with spin-squared terms in an RC spacetime whereas in GR, the spin-squared terms are missing. This implies an expanded class of meaningful solutions is possible in RC spaces compared with GR. Based on an analogy between the structure of the spin bivector and the string bivector, we have developed a string fluid stress-energy content that includes the effects of torsion and have examined a string fluid with torsion as a source for astrophysically interesting spacetimes. The stress-energy tensor that is used was derived 
variationally, and as part of the variation, the string density was identified with the torsion vector.

We applied the torsional string fluid stress-energy content to modelling a stringy atmosphere with three different metric functions. The first two choices were asymptotically the Letelier metric choice. The major difference introduced by including the torsion vector in the geometry is the presence of a transverse stress. The third model we discussed compared the field equation results to a suggestive form for a halo density profile. The model atmosphere generated from the field equations could have a boundary and the overall distance behaviour appears to be sharper than that found in the Einstein-de Sitter numerical simulations. The model was based on an extremely simple choice for the metric. A more detailed study of this behaviour is in progress.

Nucamendi and Sudarsky [37] have discussed in GR a metric for a $d=1$ monopole spacetime that includes an angular deficit. The case for strings in RC is not entirely clear [38], although the cut in the angular range and the associated conical defect is suggestive. The question of deficit angles in RC spaces is intimately connected with the existence of torsion and the idea of parallel transport [39]. This will be discussed elsewhere.

The formalism developed in this work can also be used to consider other kinds of string distributions. For example, the field equations for a string fluid of loops

$$
S^{j}=(0,0,0, S)
$$

is an interesting possibility. For this case, the off-diagonal equations $\left(G_{r \varphi}\right.$ and $\left.G_{\vartheta \varphi}\right)$ determine the torsion/string vector which is found to be

$$
S^{\varphi} S_{\varphi}=S_{o}^{2} r^{2} \sin ^{2}(\vartheta) f^{2(1-b)} .
$$

Such a string distribution would be most interestingly placed in a stationary spacetime. The vorticity in a stationary spacetime could also activate the spin part of the torsion which is linked to the trace-free part of the torsion tensor. These kinds of models could also be used to treat more realistic halo models where halo flattening is expected [40]. They might also be used to model the ring structure [41] which may have been observed in the outer portions of our own galaxy.

\section{References}

[1] Vaidya P C 1953 Nature 171260

[2] 't Hooft G 1998 Nucl. Phys. Proc. Supp. 68174

[3] 't Hooft G 1997 Plenary Lecture Notes ICMP97 (Brisbane)

[4] Glass E N and Krisch J P 1998 Phys. Rev. D 57 R5945

[5] Kar S 1997 Phys. Rev. D 554872

[6] Larsen F 1997 Phys. Rev. D 561005

[7] Letelier P S 1979 Phys. Rev. D 201294

[8] Letelier P S 1981 Nuovo Cimento B 63519

[9] Letelier P S 1987 Class. Quantum Grav. 4 L75

[10] Soleng H S 1995 Gen. Rel. Grav. 27367

[11] Burkert A 1997 Proc. Int. Workshop on Dark Matter in Astro-Particle Physics ed H V Klapdor-Kleingrothaus and Y Ramachers (Singapore: World Scientific) p 35

[12] Smalley L L and Krisch J P 1998 Comparison of string fluid dynamical models in general relativity and EinsteinCartan theory Gen. Rel. Grav. to appear

[13] Nambu Y Proc. Int. Conf. on Symmetries and Quark Models (Wayne State University)

[14] Nielson H 1970 15th Conf. on High Energy Particle Physics (Kiev)

[15] Susskind L 1970 Nuovo Cimento 69457

[16] Sherk J 1975 Rev. Mod. Phys. 47123

[17] Stachel J 1980 Phys. Rev. D 212171

[18] Rovelli C 1998 Living Reviews 
[19] Halbwachs F 1960 Theorie Relativiste des Fluide a Spin (Paris: Gauthier-Villar)

[20] Smalley L L and Krisch J P 1995 J. Math. Phys. 36778

[21] Smalley L L and Krisch J P 1996 Class. Quantum Grav. 13 L19

[22] Ray J R and Smalley L L 1982 Phys. Rev. Lett. 491059

Ray J R and Smalley L L 1982 Phys. Rev. Lett. 50 52E

[23] Ray J R and Smalley L L 1982 Phys. Rev. D 261619

[24] Ray J R 1972 J. Math. Phys. 131451

[25] Nieto J A 1995 Mod. Phys. Lett. A 103087

[26] Hehl F W, Mccrea J D, Mielke E W and Nieman Y 1995 Phys. Rep. 2581

[27] Smalley L L and Krisch J P 1992 J. Math. Phys. 331073

[28] Hammond R T 1990 Gen. Rel. Grav. 22451 Hammond R T 1991 Gen. Rel. Grav. 23973

[29] Smalley L L and Krisch J P 1997 Class. Quantum Grav. 142405

[30] Tolman R C 1939 Phys. Rev. 55364

[31] Henriksen R N and Widrew L M 1999 Mon. Not. R. Astron. Soc. 302321

[32] Navarro J F, Frenk C S and White S D M 1996 Astrophys. J. 462563

[33] Navarro J F, Frenk C S and White S D M 1997 Astrophys. J. 490493

[34] Hernquist L 1990 Astrophys. J. 356359

[35] Mann R 1997 Class. Quantum Grav. 142927

[36] Choquet-Bruhat Y, Dewitt-Demorette C and Dillard-Bleich M 1982 Analysis, Manifolds and Physics 2nd edn (Amsterdam: North-Holland) pp 300-1

[37] Nucamendi U and Sudarsky D 1997 Class. Quantum Grav. 141309

[38] Tod K P 1994 Class. Quantum Grav. 111331

[39] Smalley L L 1986 Gen. Rel. Grav. 5549

[40] Pfenniger D 1997 Proc. Int. Workshop on Dark Matter in Astro-Particle Physics ed H V Klapdor-Kleingrothaus and Y Ramachers (Singapore: World Scientific) p 54

[41] Binney J and Dehnen W 1997 Mon. Not. R. Astron. Soc. 287 L5 